\title{
50 years of newborn screening
}

\author{
Georg F. Hoffmann • Martin Lindner • J. Gerard Loeber
}

Published online: 14 February 2014

(C) SSIEM and Springer Science+Business Media Dordrecht 2014

Half a century ago Robert Guthrie published his seminal paper on newborn screening for phenylketonuria. Few of us will remember these early years and the difficulties encountered by Bob and his then few followers. The period was wonderfully summarized by him in the journal Screening in 1992 (volume 1, pages 5-15). From the 1960s to the end of the last century newborn screening progressed steadily with some technical advances and consolidated professional structures. Just before the turn of the century, highly sophisticated high-throughput multiple-analytes platforms (ELISAs, tandem MS, DNA, etc.) were developed and adapted for use in newborn screening. Particularly the applications of tandem mass spectrometry, paralleled by advances in molecular medicine, have now brought screening very much to center stage of scientific, medical and ethical issues. The Joint Meeting of the APHL Newborn Screening and Genetic Testing Symposium and the International Society for Neonatal Screening, held in Atlanta, Georgia, USA in May 2013, combined looking back and drawing from the experience of 50 years with a comprehensive coverage of today's hot topics. This issue of the journal draws on the material presented.

G. F. Hoffmann $(\bowtie) \cdot$ M. Lindner

Department of General Pediatrics, University of Heidelberg, Im

Neuenheimer Feld 430, 69120 Heidelberg, Germany

e-mail: georg.hoffmann@med.uni-heidelberg.de

M. Lindner

e-mail: martin.lindner@kgu.de

J. G. Loeber

ISNS Office, Bilthoven, The Netherlands

e-mail: gerard.loeber@gmail.com

Present Address:

M. Lindner

Department of General Pediatrics, University of Frankfurt, Theodor-

Stern-Kai 7-9, 60528 Frankfurt/Main, Germany

The World Health Organisation and the European Community have both identified orphan diseases as a major future health challenge. Among these diseases, inborn errors of metabolism are especially important because of their high morbidity and mortality, high recurrence risk in affected families, and, most importantly, emerging therapeutic options, all leading to the need to identify asymptomatic newborns by newborn screening. The introduction of cost-effective programs is the main focus of developing and third-world countries, while in the developed countries an additional focus is on questions on acceptability, regulations, management, and suitability of individual diseases for newborn screening.

The criteria for an acceptable screening program are hotly debated to date, with consolidated different results between most European countries, which in general only consider a limited number of disorders suitable for expanded MS/MS screening, and the USA with its basic recommendations of screening for $30+$ conditions with an additional $25+$ disorders some of which clearly do not meet the classical screening criteria. These discrepancies do not reflect major differences in genetic backgrounds of populations or estimated prevalences, but rather highlight different approaches to the estimation of risks and benefits for newborn screening, and just lack of evidence. We are still lacking detailed knowledge about the natural course of many diseases and their variants, information on middle- and long-term outcome after early treatment initiation, and solid data on screening projects in non-Caucasian populations. In addition to the disease panel, the spectrum of metabolites analyzed, the organization of laboratories, analytical procedures, follow-up management, proficiency, and quality control all vary considerably among countries. In addition to state-run programs there is an increasing private, sometimes internationally operating sector with a hodgepodge of disorders and methods on sale.

Conferences like that in Atlanta give us the opportunity to further develop international collaboration and to actively 
tackle these universal issues. The low incidence of some individual disorders does not and will not allow a critical evaluation even within the larger countries and thus requires a transnational approach to harmonize and optimize newborn screening. We need to develop practical case definitions for the inborn metabolic disorders included within screening to tackle the issues related to relevant phenotypic indicators as well as their heterogeneity. For tandem mass spectrometry universal metrics are necessary to allow inter-laboratory comparisons (detection rates, cut-offs, positive predictive value, false positives and negatives). International laboratory quality control schemes should be enlarged and governed. Guidelines and regulations for all aspects of the screening process are needed as well as material to inform patients and professionals, training of professionals, and, most importantly, registry-based evaluation of long-term outcome. Economic evaluations of programmes in different countries must be performed. Balancing the right to care of all patients needs to take rare disorders into account. Simple and difficult, a screening program must be adapted depending on the ethnic and genetic background, customs, social characteristics, medical environment and economic status of a country.

Despite continuing controversies, newborn screening can and does make a positive contribution at both the individual and the population level. Medium-term experiences with extended newborn screening are emerging, illustrating the substantial increase of benefit for several diseases but also the complexity of structures needed to help patients and their families. Newborn screening has become the most successful program for the secondary prevention of inherited diseases and a central issue in metabolic medicine. Bob Guthrie concluded in 1992: "While I recognize how pivotal the phenylalanine assay was for the development of newborn screening, I have always considered the filter paper blood specimen to be my most important contribution. In the simplicity of its collection ... that even molecular genetic testing can be performed...I envision many more such advances in the future."

We hope that this special issue will have its part in providing important information and stimulus to the different initiatives in countries all over the world to further advance and professionalize population newborn screening. 